\title{
G.hnem for AMI and DR
}

\section{Rossello Busquet, Ana}

Published in:

2012 International Conference on Computing, Networking and Communications (ICNC)

Link to article, DOI:

10.1109/ICCNC.2012.6167382

Publication date:

2012

Link back to DTU Orbit

Citation (APA):

Rossello Busquet, A. (2012). G.hnem for AMI and DR. In 2012 International Conference on Computing, Networking and Communications (ICNC) IEEE. https://doi.org/10.1109/ICCNC.2012.6167382

\section{General rights}

Copyright and moral rights for the publications made accessible in the public portal are retained by the authors and/or other copyright owners and it is a condition of accessing publications that users recognise and abide by the legal requirements associated with these rights.

- Users may download and print one copy of any publication from the public portal for the purpose of private study or research.

- You may not further distribute the material or use it for any profit-making activity or commercial gain

- You may freely distribute the URL identifying the publication in the public portal

If you believe that this document breaches copyright please contact us providing details, and we will remove access to the work immediately and investigate your claim 


\title{
G.hnem for AMI and DR
}

\author{
Ana Rosselló-Busquet IEEE student member \\ Networks Technology \& Service Platforms group, DTU Fotonik, \\ Technical University of Denmark, 2800 Kgs. Lyngby, Denmark \\ \{aros, joss $\} @$ fotonik.dtu.dk
}

\begin{abstract}
The power grid is evolving into the Smart Grid, by incorporating Information and Communication Technologies (ICT) and new functionalities. Some of the Smart Grid functionalities, such as Advanced Metering Infrastructure (AMI) and Demand Response (DR), require information about customers' energy consumption and production. Power Line Communication (PLC) is a candidate technology for connecting the customers and utilities as it is easy to deploy and has extensive coverage. ITU-T G.hnem standard defines a narrowband PLC, which was specially designed for Smart Grid applications. This paper proposes a PLC network architecture following the G.hnem standard, which enables bi-directional communication among customers and utilities.
\end{abstract}

\section{INTRODUCTION}

The electricity consumption in users residences has increased by $30 \%$ over the last 30 years [1]. The reason for this growth in consumption is the increase of the number of appliances that can be found in households. According to the International Energy Agency (IEA), European electricity consumption is going to increase $1.4 \%$ per year up to 2030 , unless countermeasures are taken [2].

On the other hand, the power grid has not changed significantly during the last century [3]. Therefore, the power grid has to be upgraded to solve the imminent need to efficiently generate, transmit and distribute electricity. This upgrade in the power grid will lead to the so called Smart Grid. Smart Grids will incorporate Information and Communication Technologies (ICT) to provide bidirectional communication between the different actors involved, including customers. The power grid will undergo a significant improvement to provide actors with the capability to monitor and manage the power grid more efficiently and dynamically.

Additionally, the European Commission Climate Action set three energy targets to be met by 2020 , known as the " 20 20-20" target, which reenforces the need of the Smart Grid. The first target is to reduce the European greenhouse gas emissions by $20 \%$ compared to the 1990 levels. Secondly, the energy production coming from renewable resources should be $20 \%$ of the total European generation. Finally, by improving energy efficiency the primary energy should be reduced by $20 \%$ compared with their projected levels.

The Smart Grid is still an open concept and no standard definition exists. Among the different Smart Grid definitions, the European Technology Platform (ETP) defines the Smart Grid as "an electricity network that can intelligently integrate the actions of all users connected to it generators, consumers and those that do both - in order to efficiently deliver sustainable, economic and secure electricity supplies" [3]. Another definition of the Smart Grid is made by US Department of Energy (DOE): "An automated, widely distributed energy delivery network, the Smart Grid will be characterized by a two-way flow of electricity and information and will be capable of monitoring everything from power plants to customer preferences to individual appliances. It incorporates into the grid the benefits of distributed computing and communications to deliver real-time information and enable the near-instantaneous balance of supply and demand at the device level" [4]. Even though these definitions are different, it is clear that the Smart Grid will enable a more dynamic, resilient, sustainable, efficient and adaptable grid with new capabilities that will involve the participation of the different actors in the power grid. The Smart Grid will not only handle power but also information and intelligence and will provide new functionalities which will require a robust and reliable communication network.

This article proposes a communication network for two of the Smart Grid functionalities: Advanced Metering Infrastructure (AMI) and Demand-Response (DR). The PLC network proposed collects energy consumption and generation information at the customers side and communicates it to the utility. Additionally, utilities can provide real-time price information to the consumers. The smart meter, installed at the customer's premises, collects and transmits the customers data. The energy consumption data can then be used by the service provider and utilities for grid management, outage notification, and billing purposes. DR mechanisms will be used by utilities to achieve a reduction of consumption by the customers (residential users, commercial or industrial) as a response to direct request or high electricity prices. DR will be used to reduce heavy loads in the system and to avoid demand peaks which in critical situations could lead to shortages and blackouts by sending requests through the proposed network.

The remainder of this paper is organized as follows: Section II presents the different PLC technologies and its advantages and disadvantages. The next section, Section III, provides an overview of ITU-T G.hnem standard. The G.hnem PLC network for AMI and DR is presented in Section IV. Finally, the conclusion is found in Section V.

\section{Power Line COMMUNiCATION}

Power Line Communication (PLC) is not a new technology. The first patents in PLC date back to early 1900' [5]. However, there have been new developments in this technology, that 
offers a new range of applications where PLC can be used, such as Home Automation, Internet over Broadband Power Lines and Smart Grid communications. The current PLC technologies can be divided, according to their working frequency band, into [5]:

- Ultra-Narrow Band (UNB): These technologies operate in the Ultra Low Frequency [0.3-3 kHz] band or in the upper part of the Super Low Frequency [30-300Hz] band and achieve very low data rate, around 100 bps. However, they have a very large operational range, $150 \mathrm{~km}$ or more. UNB technologies are usually proprietary and very mature.

- Narrow-Band (NB): These technologies operate in the Very Low Frequency band, Low Frequency band and Medium Frequency band $[3-500 \mathrm{kHz}]$ and can be furthermore divided into:

- Low Data Rate (LDR): It includes single carrier technologies with few kbps of data rate. The market already offers products that work in this frequency range which are usually used for home or building automation.

- High Data Rate (HDR): It includes multicarrier technologies with data rates between tens of kbps and up to $500 \mathrm{kbps}$.

- Broadband (BB): These technologies operate in the High Frequency and Very High Frequency bands (1.8$250 \mathrm{MHz}$ ) with data rates ranging from several Mbps to several hundreds Mbps. These technologies are also known as Broadband over Power Lines (BPL).

In addition, in Europe and US, governmental entities have considered PLC as a candidate technology for Smart Grid applications. Therefore, they have defined a frequency range, where this technology can operate in order to provide harmonization in the market. The Comité Européen de Normalization Electrotechnique (CENELEC) issued the EN 50065 [6] standard which allows communication over Low Voltage (LV) distribution power lines in the [3-148.5] $\mathrm{kHz}$ frequency range in Europe. Furthermore, it divides this frequency band into 4 frequency subbands:

- A band: 3-95 kHz, reserved to power utilities.

- B band: 95-125 kHz, any application.

- C band: $125-140 \mathrm{kHz}$, in home networking systems with mandatory CSMA/CA protocol.

- D band: 140-148,5 kHz, alarm and security systems.

In the US, the Federal Communications Commission (FCC) defines the [10-490] kHz frequency band for general supervision of the power system by an electric public utility [7].

\section{A. PLC Advantages}

The advantages of PLC, with focus on the AMI and DR requirements, are summarized below:

- Easy to deploy: Electricity wiring already exists and therefore no additional infrastructure is necessary. The main requirement is to deploy smart meters with PLC capabilities and introduce PLC receivers and transceivers. Depending on the distance between the users' premises and the substations, repeaters or relays may be required along the network.

- Special bandwidth reserved for utilities: CENELEC and FCC have defined a bandwidth to be used especially by utilities, which is under $500 \mathrm{kHz}$.

- Deployment costs: Traditionally, substations at the Low Voltage level are not equipped with communication capabilities [10] and deployment of new infrastructure introduces high cost for utilities. As power line cables connecting the actors involved in AMI and DR are already deployed, PLC is the only technology that has deployment costs comparable to wireless.

- High connectivity and extensive coverage: All the actors involved in AMI and DR are connected to power cables. Therefore, via a PLC network, they are connected into the AMI and DR communication network. This is an additional advantage for substations and customers in rural areas where there is usually no data communication infrastructure.

- Easily scalable: As the communication network is created, new customers and other actors can be added to the network by simply deploying a PLC transceiver.

- Redundant communication channel: AMI and DR may require redundancy in protection and control, which implies the need of available redundant communications channels. Depending on the power grid structure, which changes among countries, PLC technology can offer the necessary mesh topology for redundancy.

- Network ownership: As power line are owned by utilities a PLC network offers utilities direct and complete control of the network. This is advantageous specially on countries where telecom markets are deregulated.

- Capacity: For G.hnem the expected data rate is around $1 \mathrm{Mbps}$, which is not as high as BB-PLC or other technologies data rate. However, the US Department of Energy (DOE) has defined the data rate requirements for AMI and DR [11] to be around $100 \mathrm{kbps}$ per node and $500 \mathrm{kbps}$ for backhaul. This is based on the projections of future communications needs and the input of the different actors involved in ICT for the Smart Grid.

Furthermore, the G.hnem is a NB-PLC, which offers the following advantages over BB-PLC [10]:

- Transformer penetration: NB-PLC signals, unlike BBPLC signals, are able to "penetrate" transformers, however with a substantial SNR hit. Nevertheless, this capability depends on the transformer itself. So, if new transformers are deployed, the SNR degradation to the PLC signal should be taken into consideration.

- Ease to upgrade to future versions: Low obsolesce time for any new infrastructure is preferred. NB-PLC devices can be implemented by using a Digital Signal Processing (DSP) whereas this is not possible with BB-PLC solutions [10].

- Worldwide harmonization: FCC and CENELEC have defined a NB frequency range to be used by utilities for 
TABLE I

TECHNOLOGY COMPARISON [8], [9]

\begin{tabular}{|c|c|c|c|c|}
\hline & \multirow[t]{2}{*}{ PLC G3 } & \multirow[t]{2}{*}{ PRIME } & \multicolumn{2}{|r|}{ G.hnem } \\
\hline & & & CENELEC & FCC \\
\hline Data rate & $34 \mathrm{kbps}$ & $130 \mathrm{kbps}$ & \multicolumn{2}{|r|}{$1 \mathrm{Mbps}$} \\
\hline Typ Loop Length & $\sim 3$ & $\sim 3$ & \multirow{2}{*}{\multicolumn{2}{|c|}{$\begin{array}{c}\sim 3-4 \\
\text { Ipv6, IPv4, Ethernet }\end{array}$}} \\
\hline Transport Layer & IPv6 & IPv4, IEC 62056, IEC 61334-4-32 & & \\
\hline Working Bandplans & $3-95 \mathrm{kHz}$ and $150-490 \mathrm{kHz}$ & $3-95 \mathrm{kHz}$ & $35-143 \mathrm{kHz}$ & $34-478 \mathrm{kHz}$ \\
\hline \multicolumn{5}{|l|}{ OFDM } \\
\hline - FFT size & 256 & 512 & 256 & 512 \\
\hline - Sampling frequency & $400 \mathrm{kHz}$ & $250 \mathrm{kHz}$ & $400 \mathrm{kHz}$ & $1600 \mathrm{kHz}$ \\
\hline - Length cyclic prefix & 30 & 48 & 20 or 32 & 40 or 64 \\
\hline - Windowing size & 8 samples & - & 8 samples & 16 samples \\
\hline - subcarrier spacing & $1.5625 \mathrm{kHz}$ & $488 \mathrm{~Hz}$ & $1.5625 \mathrm{kHz}$ & $3.125 \mathrm{kHz}$ \\
\hline Forward Error Correction & Reed Solomon code and convolutional code & convolutional code & \multicolumn{2}{|c|}{ Reed Solomon code and convolutional code } \\
\hline Modulation & DBPSK, DQPSK & DBPSK, DQPSK, D8PSK & \multicolumn{2}{|c|}{$\begin{array}{l}\text { BPSK, QPSK, 16-QAM } \\
\text { constellation mapping for } 3 \text { bits is under study }\end{array}$} \\
\hline
\end{tabular}

Smart Grid functionalities. Furthermore, some countries, have prohibited the use of frequencies above $2 \mathrm{MHz}$, which is the frequency band for BB-PLC, in outdoor environments [10].

- Optimized design: BB-PLC solutions, such as IEEE 1901 [12] or ITU-T G.hn, were originally developed for home networking and broadband Internet access and not for Smart Grid functionalities. However, G.hnem, and also IEEE 1901.2 [13] were specially designed for Smart Grid functionalities, such as AMI and DR.

\section{B. PLC Disadvantages}

NB-PLC, among them G:hnem, poses the following disadvantages:

- New Standard: Even though some PLC technologies have passed the experimental phase and are mature technologies. G.hnem has just started their final stages of standardization and has not reach the mass market penetration.

- Interference: G.hnem and IEEE 1901.2 work in the same frequency band and power lines are a shared medium, which can cause interoperability problems. However, interference among devices can be solved by using coexistence mechanisms, such as those defined in ITU-T G.9972 recommendation [14].

- Harsh and noisy channel: The power line medium is difficult to model, it is frequency selective, time-varying, and is impaired by colored background noise and impulsive noise.

- Grid structure: Creating a PLC network for AMI and DR can be challenging as the grid structure differs from country to country and also within a country and more than one utility may be providing electricity to customers.

\section{G.HNEM}

ITU-T G.hnem standard [15] can be used to implement the access network for data collection for AMI and DR and send DR requests. This standard was designed specially to support Smart Grid applications, such as AMI and DR. It is composed of two recommendations G.9955, "Physical layer specification" [16] and G.9956, "Data link layer specification" (DLL) [17] for Narrowband Orthogonal Frequency-Division Multiplexing (OFDM) Power Line Communication.

G.hnem recommendation is based on PLC G3 [18] and PRIME [19], which are both public and open and have already undertaken field trials [20], [21]. These technologies are classified as Narrowband High Data Rate Power Line Communication (NB-HDR PLC) using OFDM.

A comparison of PLC G3, Prime and G.hnem can be found in Table I. In the table, CENELEC and FCC frequency ranges are distinguished, as the G.hnem defines different OFDM parameters for transmission. The main advantage of the ITU-T recommendation is the higher data rate, up to $1 \mathrm{Mbps}$.

\section{A. G.hnem Network Architecture}

A G.hnem network is divided into one or more domains. A domain is a logical group of nodes, therefore domains may physically, fully or partially, overlap. The domains are identified by a 16-bits Domain ID, which is unique inside the G.hnem network. Furthermore, a G.hnem network may contain alien domains, which are any group of non-G.hnem nodes connected to the G.hnem network through a bridge node. A G.hnem network can consist of up to 65535 domains $\left(2^{16}-1\right)$ and each domain can contain 32768 nodes $\left(2^{15}\right)$. Each node is identified by a unique 16-bits Node ID inside a domain. In a G.hnem network, nodes can be extended to provide none, one or more of the following capabilities:

- Global Master (GM): Each network has a GM which coordinates the operation, resources, priorities and operational characteristics, of different domains in the network.

- Domain Master (DM): Each domain shall contain a domain master (DM), which manages and coordinates the operation of all nodes in its domain. It is not required that all nodes are domain master capable. However, there has to exactly one domain master at all times.

- Inter-Domain Bridge (IDB): IDB nodes enable communication between nodes belonging to different domains inside the same network. 
- Layer 3 Bridge (L3 bridge): The main function of this type of bridge is to connect an alien domain with a G.hnem domain and to coordinate both domains to avoid mutual interference. However, these functionalities are beyond G.hnem scope.

- Relay node: Relays nodes have the capability to be used when direct communication among nodes is not possible.

- Domain Access Point (DAP): This type of node is not compulsory in a G.hnem domain, it is only found in a domain if the domain works in centralized communication mode. The DAP receives frames from all the nodes in the domain and forwards them to the corresponding destination node.

In a G.hnem network, nodes can communicate with each other directly or via one or more other nodes. Therefore, mesh, star and tree topologies are supported by G.hnem. In the G.hnem standard, 3 types of communication are defined:

- Peer-to-peer communications (P2P): Nodes can only communicate with each other directly. If a node wants to communicate with a node outside the domain, frames are sent to the corresponding IDB.

- Centralized Mode (CM): This type of communication requires a domain access point (DAP). Hence, any node in the domain that wants to communicate with another node has to do it through the DAP node. In general, the DAP node is also the domain master, however, this is not compulsory. If a node cannot reach the DAP with direct communication, nodes acting as relays are used. Communication between nodes is not allowed in the case of DAP failure.

- Unified Mode (UM): Nodes in a domain can communicate directly or via relays. IDB receive all the frames that have to be send to nodes outside the domain.

\section{B. G.hnem Recommendations}

As stated before G.hnem standard consists of two recommendations: G.9955 and G.9956.

G.9955 describes the PHY layer and defines its parameters. The PHY layer main functionalities are: create PHY frames from MAC Protocol Data Units (MPDU), encode the PHY frames, and modulate the OFDM symbols. The main parameters of this layer are included in Table I.

G.9956 describes the DLL and defines its parameters. The DLL layer is in charge of creating the MPDU from the upper layer frames, which can be, for instance, IPv6 or IPv6 6LoWPAN compressed. Nodes can also exchange Link Control Data Units (LCDU), which are management frames exchange between LLCs. G.hnem also supports Quality of Service (QoS) by classifying frames into 4 different priorities. However, the highest priority should only be used for emergency data transfers only.

Security is provided optionally by encryption of the communicated frames. The encryption method specified in this standard is based on the Advanced Encryption Standard (AES) and Counter Mode with Cipher Block Chaining Message Authentication Code protocol (CCMP). The standard also

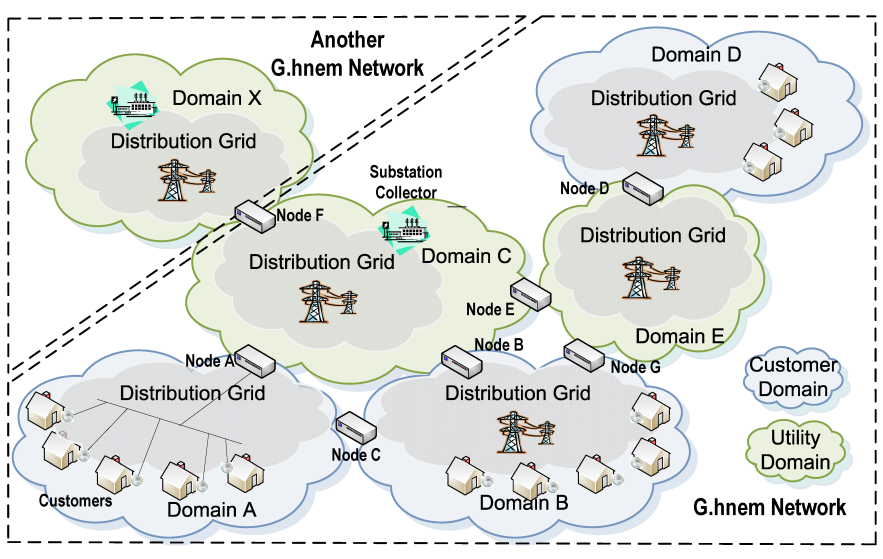

Fig. 1. PLC Network Architecture

defines a set of Authentication and Key Management (AKM) procedures for authentication, generation and distribution of encryption keys between nodes. However, this is still under development.

The G.hnem standard also defines procedures for retransmission and acknowledgement of frames. Media Access Control is also defined in terms of contention periods, where higher priority frames are more likely to gain access.

\section{AMI AND DR ARCHITECTURE}

Advanced Metering Infrastructure (AMI) allows utilities to collect metering information via a communication network with customers and to analyze energy consumption data for grid management, outage notification, and billing purposes.

Moreover, Demand-Response (DR) is the reduction of consumption of electricity by customers in response to an increase in the price of electricity or due to a direct request from the utility. However, DR mechanism need information about the customers consumption and generation.

The collected data by the smart meter, which acts as an access point, can be transferred to the utility using G.hnem Power Line Communication (PLC). Furthermore, DR requests and electricity price can also be sent to the customers through the PLC network.

The proposed G.hnem network is shown in Fig. 1. Due to the fact that the grid network topology varies from country to country, it is hard to find a general example of the electrical distribution network topology. However, most electrical distribution networks are radial with a large amount of end nodes (customers). Therefore, the domains are represented as cloud in Fig. 1. The domain $\mathrm{X}$ in the figure belongs to a different G.hnem network. Therefore, node F acts as a bridge between these two G.hnem networks.

The proposed network aims to gather data from the end nodes, collecting it at the utility substation. As this PLC network is a bidirectional network, the substation can send requests to the customers to reduce their consumption and other DR messages. The network is divided into two types of domains: Customer Domains (CD) and Utility Domains (UD). These two domains have been classified according to their functionality. 


\section{A. Customer Domain}

Customers domains are the domains where customers are connected to communicate with utilities, such as Domain A, B and C. The Domain Master (DM) is located in the distribution network and it is owned and control by the utility. This enables the utilities to have control over the communication network. The DMs for the customers domains in Fig. 1 are node A, node B and node D. As the exchange of information in these domains is between customers and the Domain Master, centralized communication mode has been proposed. The DM, in this case, will also act as DAP and IDB. The DM will gather the customers information and forward it to the Global Master (GM), in this case a substation. If the substation is situated far away from the customers, for instance in rural areas, the collected data may have to bypass one or more utility domains.

In this domain the customers are connected to the G.hnem network via the smart meter. Some of these smart meters may act as relay nodes when direct communication between another customer node and the DM is not possible. Furthermore, the domain may have more than one IDB (node $\mathrm{C}$ and $\mathrm{G}$ in the Fig. ??) to communicate with other customers domains.

\section{B. Utility Domain}

Utilities domains are domains where the communication is among utilities' nodes, such as Domain C, E and X. The DM chosen for these domains is a node within the domain that may or may not act as a IDB. In this type of domain communication mainly occurs with the GM to collect the customer's data. However, the communication among all nodes in the domain is an interesting feature to enable a more distributed management. Therefore, unified mode communication is proposed. Utility domains may have more than one IDB to communicate with other utility domains or customer domains. Communication with other G.hnem networks is also possible and will enable full coordination in the distribution grid.

As all the nodes involved in this type of domain are owned by the utility, the utility has full control of the communication network.

\section{CONCLUSiON}

AMI and DR require a bi-directional communication network to collect customers consumption and generation data. However, the present power grid does not have the communication infrastructure to support this communication and new network architectures have to be deployed. In this paper, a network architecture for AMI and DR using PLC has been proposed. This network aims to gather data from the customers and collected at the utility's substation. Furthermore, through this network, utilities can communicate real-time price information and send DR requests to customers.

PLC technologies have been presented and their advantages and disadvantages have been discussed. ITU-T G.hnem standard defines a narrow-band PLC, which was specially designed for Smart Grid applications. Section III describes the G.hnem network architecture, its main characteristics and compares it to G3 and PRIME. In the final section, the G.hnem network architecture for AMI and DR is proposed and presented in detail.

\section{REFERENCES}

[1] BeyWatch Consortium, "D2.1:Service Requirement Specification," 2009.

[2] European Commission, European Technology Platform SmartGrids Vision and Strategy for Europe's Electricity Networks of the Future. Office for Official Publications of the European Communities, 2006.

[3] European Technology Platform SmartGrids for the Electricity networks of the Future, "What is a Smart Grid? Definition." [Online]. Available: http://www.smartgrids.eu/?q=node/163 Accessed: 25/06/2011

[4] Department of Energy, "The Smart Grid An Introduction," U.S. Department of Energy, 2008.

[5] S. Galli, A. Scaglione, and Z. Wang, "For the grid and through the grid: The role of power line communications in the smart grid," Proceedings of the IEEE, vol. 99, no. 6, pp. $998-1027$, june 2011.

[6] CENELEC, "Signaling on low-voltage electrical installations in the frequency range $3 \mathrm{kHz}$ to $148.5 \mathrm{kHz}$ Tech. Rep. EN 50 065-1," 1991.

[7] Federal Communications Commission, "Inquiry Regarding Carrier Current Systems, including Broadband over Power Line Systems," ITU-T, Tech. Rep. FCC 03-100, 2003.

[8] V. Oksman, "New ITU-T Recommendations for Smart Grid in-home and access communications." Presented at ETSI Smart Grid Workshops Sophia Antipolis, France, 2011.

[9] M. Hoch, "Comparison of ple g3 and prime," in Power Line Communications and Its Applications (ISPLC), 2011 IEEE International Symposium on, april 2011, pp. $165-169$

[10] S. Galli, A. Scaglione, and Z. Wang, in Smart Grid Communications (SmartGridComm), 2010 First IEEE International Conference on, title $=$ Power Line Communications and the Smart Grid, oct. 2010, pp. $303-308$.

[11] "Communications Requirements of the Smart Grid Technologies," Department of Energy (DOE), Tech. Rep., 2010.

[12] "IEEE Draft Standard for Broadband over Power Line Networks: Medium Access Control and Physical Layer Specifications," IEEE P1901/D4.01, July 2010, 2010.

[13] "IEEE Draft Standard for Low Frequency (less than $500 \mathrm{kHz}$ ) Narrow Band Power Line Communications for Smart Grid Applications[4] Recommendation ITU-T G.9972 (2010), Coexistence mechanism for wireline home networking transceivers." IEEE P1901/D4.01, July 2010, 2011.

[14] "Coexistence mechanism for wireline home networking transceivers," 2010.

[15] "G.hnem project Architecture and Current status," 2010. [Online]. Available: http://ftp.tiaonline.org/TR-50/TR50_main/Public/20100310_Denver_CO/TR50-20100310-006_G hnem project - architecture and current status.pdf Accessed: 25/06/2011

[16] S. Galli, "Draft Recommendation ITU-T G.9955," ITU-T, Tech. Rep. TD 413 Rev.1 (PLEN/15), 2011. [Online]. Available: http://www.itu.int/itut/workprog/wp_item.aspx?isn=7701 Accessed: 25/06/2011

[17] _ , "Draft Recommendation ITU-T G.9956," ITU-T, Tech. Rep. TD 412 Rev.1 (PLEN/15), 2011. [Online]. Available: http://www.itu.int/itut/workprog/wp_item.aspx?isn=7876 Accessed: 25/06/2011

[18] MAXIM. (2011, june) G3-plc. [Online]. Available: http://www.maximic.com/products/powerline/g3-plc/ Accessed: 25/06/2011

[19] PRIME Alliance. [Online]. Available: http://www.prime-alliance.org Accessed: 25/06/2011

[20] K. Razazian, A. Kamalizad, M. Umari, Q. Qu, V. Loginov, and M. Navid, "G3-plc field trials in u.s. distribution grid: Initial results and requirements," in Power Line Communications and Its Applications (ISPLC), 2011 IEEE International Symposium on, april 2011, pp. 153 -158 .

[21] A. Aruzuaga, I. Berganza, A. Sendin, M. Sharma, and B. Varadarajan, "Prime interoperability tests and results from field," in Smart Grid Communications (SmartGridComm), 2010 First IEEE International Conference on, oct. 2010, pp. $126-130$. 